\title{
Early Ultraviolet Observations of Type IIn Supernovae Constrain the Asphericity of Their Circumstellar Material
}

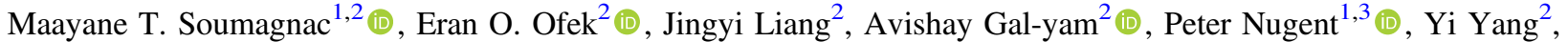 \\ S. Bradley Cenko ${ }^{4,5}$ (i) , Jesper Sollerman ${ }^{6}$ (i), Daniel A. Perley ${ }^{7}$ (D) Igor Andreoni $^{8}$ (i), Cristina Barbarino ${ }^{6}$, Kevin B. Burdge $^{8}$ (i), \\ Rachel J. Bruch ${ }^{2}$ (1), Kishalay De ${ }^{8}$ (D), Alison Dugas ${ }^{8}$, Christoffer Fremling ${ }^{8}$ (D), Melissa L. Graham ${ }^{9}$ (i), Matthew J. Hankins ${ }^{8}$ (ID), \\ Nora Linn Strotjohann ${ }^{2}$ (i), Shane Moran ${ }^{10,11}$, James D. Neill ${ }^{8}$ (10), Steve Schulze ${ }^{2}$ (10), David L. Shupe ${ }^{13}$ (10) Brigitta M. Sipőcz ${ }^{9}$,

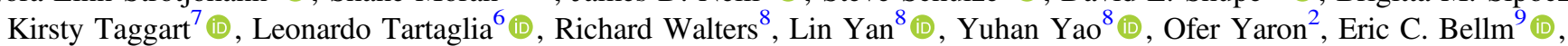

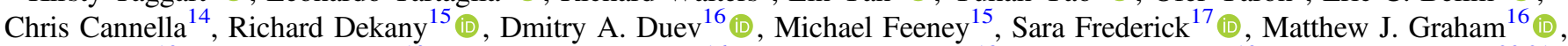

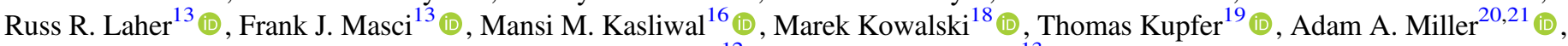 \\ Mickael Rigault ${ }^{12}$ (10), and Ben Rusholme ${ }^{13}$ \\ ${ }^{1}$ Lawrence Berkeley National Laboratory, 1 Cyclotron Road, Berkeley, CA 94720, USA; mtsoumagnac@lbl.gov \\ ${ }^{2}$ Department of Particle Physics and Astrophysics, Weizmann Institute of Science, Rehovot 76100, Israel \\ ${ }^{3}$ Department of Astronomy, University of California, Berkeley, CA 94720-3411, USA \\ ${ }^{4}$ Astrophysics Science Division, NASA Goddard Space Flight Center, MC 661, Greenbelt, MD 20771, USA \\ ${ }^{5}$ Joint Space-Science Institute, University of Maryland, College Park, MD 20742, USA \\ ${ }^{6}$ The Oskar Klein Centre, Department of Astronomy, Stockholm University, AlbaNova, SE-10691 Stockholm, Sweden \\ Astrophysics Research Institute, Liverpool John Moores University, 146 Brownlow Hill, Liverpool L3 5RF, UK \\ ${ }^{8}$ California Institute of Technology, 1200 East California Boulevard, MC 278-17, Pasadena, CA 91125, USA \\ 9 DIRAC Institute, Department of Astronomy, University of Washington, 3910 15th Avenue NE, Seattle, WA 98195, USA \\ ${ }^{10}$ Tuorla Observatory, Department of Physics and Astronomy, FI-20014, University of Turku, Finland \\ ${ }^{11}$ Nordic Optical Telescope, Apartado 474, E-38700 Santa Cruz de La Palma, Spain \\ ${ }^{12}$ Universite Clermont Auvergne, CNRS/IN2P3, Laboratoire de Physique de Clermont, F-63000 Clermont-Ferrand, France \\ ${ }^{13}$ IPAC, California Institute of Technology, 1200 E. California Blvd, Pasadena, CA 91125, USA \\ ${ }^{14}$ Department of Electrical and Computer Engineering, Duke University, Durham, North Carolina 27708, USA \\ ${ }^{5}$ Caltech Optical Observatories, California Institute of Technology, Pasadena, CA, USA \\ ${ }^{16}$ Division of Physics, Mathematics and Astronomy, California Institute of Technology, Pasadena, CA 91125, USA \\ ${ }^{17}$ Department of Astronomy, University of Maryland College Park, College Park, MD 20742, USA \\ ${ }^{18}$ DESY, 15738 Zeuthen, Germany Institut for Physik, Humboldt-Universitt zu Berlin, D-12489 Berlin, Germany \\ ${ }^{19}$ Kavli Institute for Theoretical Physics, University of California, Santa Barbara, CA 93106, USA \\ ${ }^{20}$ Center for Interdisciplinary Exploration and Research in Astrophysics (CIERA) and Department of Physics and Astronomy, Northwestern University, 2145 \\ Sheridan Road, Evanston, IL 60208, USA \\ ${ }^{21}$ The Adler Planetarium, Chicago, IL 60605, USA \\ Received 2020 January 9; revised 2020 April 30; accepted 2020 May 18; published 2020 August 11
}

\begin{abstract}
We present a survey of the early evolution of 12 Type IIn supernovae (SNe IIn) at ultraviolet and visible light wavelengths. We use this survey to constrain the geometry of the circumstellar material (CSM) surrounding SN IIn explosions, which may shed light on their progenitor diversity. In order to distinguish between aspherical and spherical CSM, we estimate the blackbody radius temporal evolution of the SNe IIn of our sample, following the method introduced by Soumagnac et al. We find that higher-luminosity objects tend to show evidence for aspherical CSM. Depending on whether this correlation is due to physical reasons or to some selection bias, we derive a lower limit between $35 \%$ and $66 \%$ for the fraction of SNe IIn showing evidence for aspherical CSM. This result suggests that asphericity of the CSM surrounding SNe IIn is common-consistent with data from resolved images of stars undergoing considerable mass loss. It should be taken into account for more realistic modeling of these events.
\end{abstract}

Unified Astronomy Thesaurus concepts: Supernovae (1668)

\section{Introduction}

Type IIn supernovae (SNe IIn) show prominent and narrowto-intermediate width Balmer emission lines in their spectra (Schlegel 1990; Filippenko 1997; Smith 2014; Gal-Yam 2017). This specificity is thought to be the signature of photoionized and dense, hydrogen-rich, circumstellar medium (CSM) which is ejected from the $\mathrm{SN}$ progenitor prior to its explosive death. Because they are the signature of an external physical phenomenon rather than of any intrinsic property of the explosion, these narrow lines may appear in the spectra of many $\mathrm{SNe}$ at some point during their evolution. As a result, the Type IIn class of SNe is a heterogeneous category of objects. Depending on the spatial distribution and physical properties of the CSM surrounding the explosion, the characteristic narrow Balmer lines may persist for days ("flash spectroscopy," GalYam et al. 2014; Khazov et al. 2016; Yaron et al. 2017), weeks (e.g., SN 1998S, Li et al. 1998; Fassia et al. 2000, 2001; SN 2005gl, Gal-Yam et al. 2007; SN 2010mc, Ofek et al. 2013b), or years (e.g., SN 1988Z, Danziger \& Kjaer 1991; Stathakis \& Sadler 1991; Turatto et al. 1993; van Dyk et al. 1993; Chugai \& Danziger 1994; Fabian \& Terlevich 1996; Aretxaga et al. 1999; Williams et al. 2002; Schlegel \& Petre 2006; Smith et al. 2017; SN 2010j1, Patat et al. 2011; Stoll et al. 2011; Gall et al. 2014; Ofek et al. 2014).

Observing $\mathrm{SNe}$ IIn at ultraviolet (UV) wavelengths is interesting for several reasons. First, an important ingredient of the physical picture governing $\mathrm{SNe}$ IIn explosions-the 
collisionless shock propagating in the CSM after the shock breakout (Ofek et al. 2010) - is predicted to radiate most in the UV and X-rays (Katz et al. 2011; Murase et al. 2011, 2014; Chevalier \& Irwin 2012). Observing the explosion at these wavelengths has the potential to unveil precious information about the explosion mechanism and the CSM properties (e.g., Ofek et al. 2013a). In particular, it may provide a much better estimate of the bolometric luminosity of the event.

Second, UV observations can help constrain the geometrical distribution of the CSM, which is closely related to the massloss processes occurring before the explosion, and probe the nature of the progenitors of this type of events.

Although observations of SNe IIn are usually analyzed within the framework of spherically symmetric models of CSM, resolved images of stars undergoing considerable mass loss (e.g., $\eta$ Carinae; Davidson \& Humphreys 1997, 2012), some of which are probably SN IIn progenitors (Gal-Yam et al. 2007; Gal-Yam \& Leonard 2009), as well as polarimetric observations (Leonard et al. 2000; Hoffman et al. 2008; Wang \& Wheeler 2008; Reilly et al. 2017), suggest that asphericity should be taken into account for more realistic modeling. Asphericity of the CSM has recently been invoked to interpret the spectrocopic and spectropolarimetric observations of the Type IIn SN 2012ab (Bilinski et al. 2018) and SN 2009ip (Levesque et al. 2014; Mauerhan et al. 2014; Smith et al. 2014; Reilly et al. 2017).

In Soumagnac et al. (2019b), we showed that the light curve of the luminous Type IIn SN PTF $12 \mathrm{glz}$ may be interpreted as evidence of an aspherical CSM. While the spectroscopic analysis is consistent with an opaque CSM obstructing our view of any growing structure, $r_{\mathrm{BB}}$ - the radius of the deepest transparent emitting layer-grows by an order of magnitude, at a speed of $\sim 8000 \mathrm{~km} \mathrm{~s}^{-1}$. To explain this tension, we considered a simple aspherical structure of CSM: a threedimensional slab, infinite in two dimensions ( $x$ and $y$ axis) and perpendicular to the line of sight (z-axis). We modeled the radiation from an explosion embedded in a slab of CSM by numerically solving the radiative diffusion equation in a slab with different density profiles: $\rho=$ Const., $\rho \propto|z|^{-1}$ and a wind density profile $\rho \propto z^{-2}$. Although this model is simplistic, it allows recovery of the peculiar growth of the blackbody radius $r_{\mathrm{BB}}$ observed in the case of PTF $12 \mathrm{glz}$, as well as the decrease of its blackbody temperature $T_{\mathrm{BB}}$.

This allowed us to derive a criterion for asphericity: a fast increase of $r_{\mathrm{BB}}$ can be interpreted as the signature of non-spherical CSM, if it is observed while the CSM is still optically thick. This is because the approximately stationary CSM is obscuring the expanding SN ejecta, and explaining an expanding emitting region due to photon diffusion in the CSM requires a nonspherical CSM configuration. In this paper, we assemble a sample of SNe IIn, to which we apply this criterion in order to estimate the fraction of SNe IIn showing evidence for non-spherical CSM.

Several samples of SNe IIn have been gathered and studied so far. Among them, the sample by Kiewe et al. (2012), consists of four SNe IIn observed by the Caltech Core-Collapse Project with the $1.5 \mathrm{~m}$ robotic telescope at the Palomar Observatory (P60; Cenko et al. 2006) using Johnson-Cousins $B V R I$ filters. They studied the light-curve features and derived the progenitor star wind velocities. The sample by Taddia et al. (2013) consists of five SNe IIn observed by the Carnegie Supernova Project (Hamuy et al. 2006) at visible light and near-infrared wavelengths, and was used to derive mass-loss parameters. The sample by Ofek et al. (2014) consists of 19 SNe IIn observed by the Palomar Transient Factory (Law et al. 2009; Rau et al. 2009) and its extension, the intermediate PTF (iPTF) using the PTF $R$-band filter. It allowed us to exhibit a possible correlation between the $r$-band rise time and peak luminosity of SNe IIn and to derive lower limits on the shockbreakout velocity, supporting the idea that early-time light curves of SNe IIn are caused by shock breakout in a dense CSM. The sample by Nyholm et al. (2020) consists of 42 objects with observations from PTF and $\mathrm{PTT}$, and was used for an in-depth study of their light-curve properties. They found only a weak such correlation. de la Rosa et al. (2016) collected Swift UV observations of 10 SNe IIn observed between 2007 and 2013 (eight of which were post-peak) and studied, e.g., their blackbody properties. To our knowledge, no systematic and planned survey of the early phase of SNe IIn in the UV has been performed so far. In this paper, we present a sample of 12 SNe IIn detected and observed by the Zwicky Transient Facility (ZTF; Bellm et al. 2019; Graham et al. 2019) and followed up in the UV by the Neil Gehrels Swift Observatory (Swift) space telescope (Gehrels et al. 2004), using Swift's Ultraviolet/Optical Telescope (UVOT; Roming et al. 2005; Poole et al. 2008; Breeveld et al. 2011).

We present the abovementioned observations in Section 2. In Section 3, we present an analysis of these observations. Section 4 is dedicated to constraining the fraction of SNe IIn exploding into aspherical CSM. We summarize our main results in Section 5.

\section{Observations and Data Reduction}

In this section, we present the ZTF and Swift observations of the 12 SNe IIn of our sample.

\subsection{Discovery}

All 12 SNe IIn were detected by the ZTF automatic pipeline as potential transients in the data from the ZTF camera mounted on the $1.2 \mathrm{~m}$ Samuel Oschin telescope (P48, Rahmer et al. 2008). A duty astronomer reviewing the ZTF alert stream (Patterson et al. 2019) via the ZTF GROWTH Marshal (Kasliwal et al. 2019). The coordinates, redshift and distance modulus of all objects are summarized in Table 1. The Milky Way extinction was deduced from Schlafly \& Finkbeiner (2011) using the extinction curves of Cardelli et al. (1989).

\subsection{Selection Criterion}

Since the beginning of its operation, ZTF has found several spectroscopically confirmed SNe IIn per month. However, applying the criterion for asphericity from Soumagnac et al. (2019b) depends on our ability to measure the evolution of $r_{\mathrm{BB}}$ - the effective blackbody radius - at the time when the CSM is still optically thick and obstructing our view of any expanding material. We selected only $\mathrm{SNe}$ IIn that were spectroscopically confirmed while still on their rise. This selection criterion was motivated by three reasons: (1) the spectrum of the SNe IIn in the early phase is still well described by a blackbody spectrum; (2) the rise of the optical light curve provides gives a better handle the evolution of $r_{\mathrm{BB}}$ than the peak phase; (3) we assumed that rising SNe IIn are young enough to 
Table 1

Summary of Observational Parameters

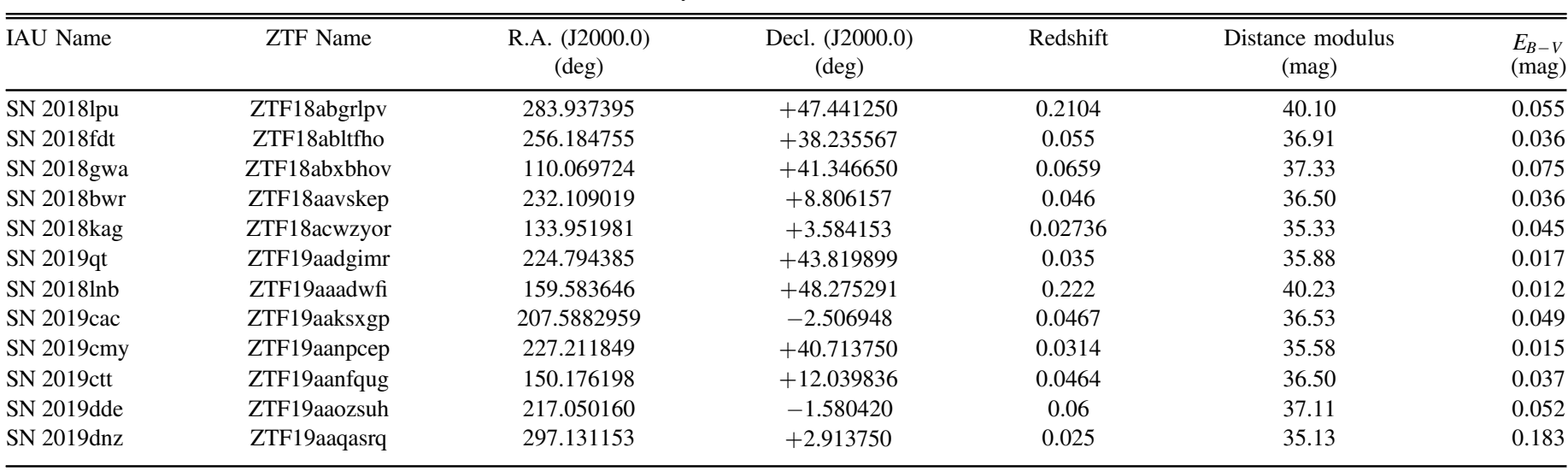

Note. The three first SNe are those for which we were unable to secure enough spectroscopic data in order to include them in our analysis of the CSM geometry (see Section 3.3). SN 2018lpu was discovered and classified by the ZTF survey; SN 2018fdt was discovered by the ATLAS survey on 2018 August 14 as ATLAS18tuy (Tonry et al. 2018b), also detected by Gaia surveys as Gaia18chl, classified by ZTF (Fremling et al. 2018a); and SN 2018gwa was discovered (Fremling 2018) and classified (Fremling et al. 2018b) by ZTF, also detected by Gaia on 2018 October 5 as Gaia18cxl. The rest of the SNe in the table are all included in our analysis of the CSM geometry. SN 2018bwr was discovered by the ATLAS survey on 2018 May 21 as ATLAS18ppb (Tonry et al. 2018a), also detected by PS1 and Gaia surveys as PS18aau and Gaia18bpl, classified by ZTF (Fremling \& Sharma 2018); SN 2018kag was discovered by the ASAS-SN survey on 2018 December 17 as ASASSN18abt and classified by Prentice et al. (2018); SN 2019qt was discovered (Nordin et al. 2019a) and classified (Payne et al. 2019) by ZTF, also detected by ATLAS, Gaia, and PS1 as ATLAS19btl, Gaia19aid, and PS19ahv; SN 2018lnb was discovered and classified by ZTF (Fremling et al. 2019a); SN 2019cac was discovered and classified by ZTF (Fremling 2019a), also detected by ATLAS and PS1 as ATLAS19doj and PS19ym; SN 2019cmy was discovered (Nordin et al. 2019b) and classified (Fremling et al. 2019b) by ZTF, also detected by ATLAS as ATLAS19elx; SN 2019ctt was discovered by ZTF (Nordin et al. 2019c) and classified by SCAT (Tucker et al. 2019); SN 2019dnz was discovered by ZTF (Fremling 2019b) and classified by TCD (Prentice et al. 2019), also detected by ATLAS as ATLAS19hra; SN 2019dde was discovered by ZTF, classified by ZTF (Fremling et al. 2019c) and (Cartier et al. 2019), also detected by MASTER and PS1 as MASTER OT $\mathrm{J} 142812.05-013615.2$ and PS19aaa.

allow us to take several Swift observations and still be in the regime where expanding material has not reached optically thin areas of the CSM. The initial classification, within ZTF, was triggered as part of a variety of programs: the Redshift Completeness Factor (RCF; Fremling et al. 2019d) program, the Census of the Local universe program (De et al. 2019), the Superluminous Supernovae program, the Rapidly Evolving Transients program, and the Science Validation program or the SNe IIn program. Some of these objects were first reported and classified by other surveys; see Table 1 for details.

\subsection{Photometry}

All the light curves are shown in Figure 1. The photometry is reported in electronic Table 2 and is available via WISeREP. ${ }^{22}$

Photometry was obtained using the ZTF camera mounted on the P48 telescope, through the P48 $r$ and $g$ filters. Data were obtained with a cadence of about 1-3 days, to a limiting AB magnitude of $r=20.5 \mathrm{mag}$ and $g=21 \mathrm{mag}$. The P48 data were automatically reduced using the ZTF pipeline (Masci et al. 2019), using the image subtraction algorithm ZOGY by Zackay et al. (2016).

The robotic $1.52 \mathrm{~m}$ telescope at Palomar (P60; Cenko et al. 2006) was used with a $2048 \times 2048$-pixel "Rainbow" CCD camera (Ben-Ami et al. 2012; Blagorodnova et al. 2018) and $g^{\prime}, r^{\prime}, i^{\prime}$ SDSS filters. Data reduction of the P60 data was performed using the FPipe pipeline (Fremling et al. 2016), using the image subtraction algorithm by Zackay et al. (2016).

For several SNe IIn discussed in this work, We acquired multi-band images with the optical imager (IO:O) on the

\footnotetext{
22 https://wiserep.weizmann.ac.il
}

Liverpool Telescope (LT; Steele et al. 2004). Image reductions were provided by the basic $\mathrm{IO}: \mathrm{O}$ pipeline and image subtraction was performed versus PS1 $(g, r, I, z$ bands) or SDSS ( $u$ band) reference imaging, following the techniques of Fremling et al. (2016). PSF photometry was performed relative to PS1/SDSS photometric standards.

The Swift UVOT data were retrieved from the NASA Swift Data Archive ${ }^{23}$ and reduced using standard software distributed with HEAsoft version $6.26 .{ }^{24}$ Photometry was measured using the FTOOLSs uvotimsum and uvotsource with a $5^{\prime \prime}$ circular aperture.

None of the SNe IIn in our sample were detected with the Swift XRT camera.

\subsection{Spectroscopy}

Optical spectra of all SNe were obtained using the telescopes and spectrographs listed in Table 3. The spectra were used to determine the redshift from the narrow host lines $(\mathrm{H} \alpha)$. All the spectra were corrected for Galactic extinction as deduced from Schlafly \& Finkbeiner (2011), using Cardelli et al. (1989) extinction curves.

All spectra are shown in Figure 2 and are available from WISeREP. In the following, we summarize the reduction procedures applied for each spectrum. All spectroscopic observations were calibrated in the following way: since we have contemporaneous P48 $r$-band data, all spectra were scaled so that their synthetic photometry matches the P48 $r$-band value.

\footnotetext{
$\overline{23}$ https://heasarc.gsfc.nasa.gov/cgi-bin/W3Browse/swift.pl

${ }^{24}$ https://heasarc.gsfc.nasa.gov/docs/software/heasoft/, http://heasarc.gsfc.nasa. gov/ftools
} 

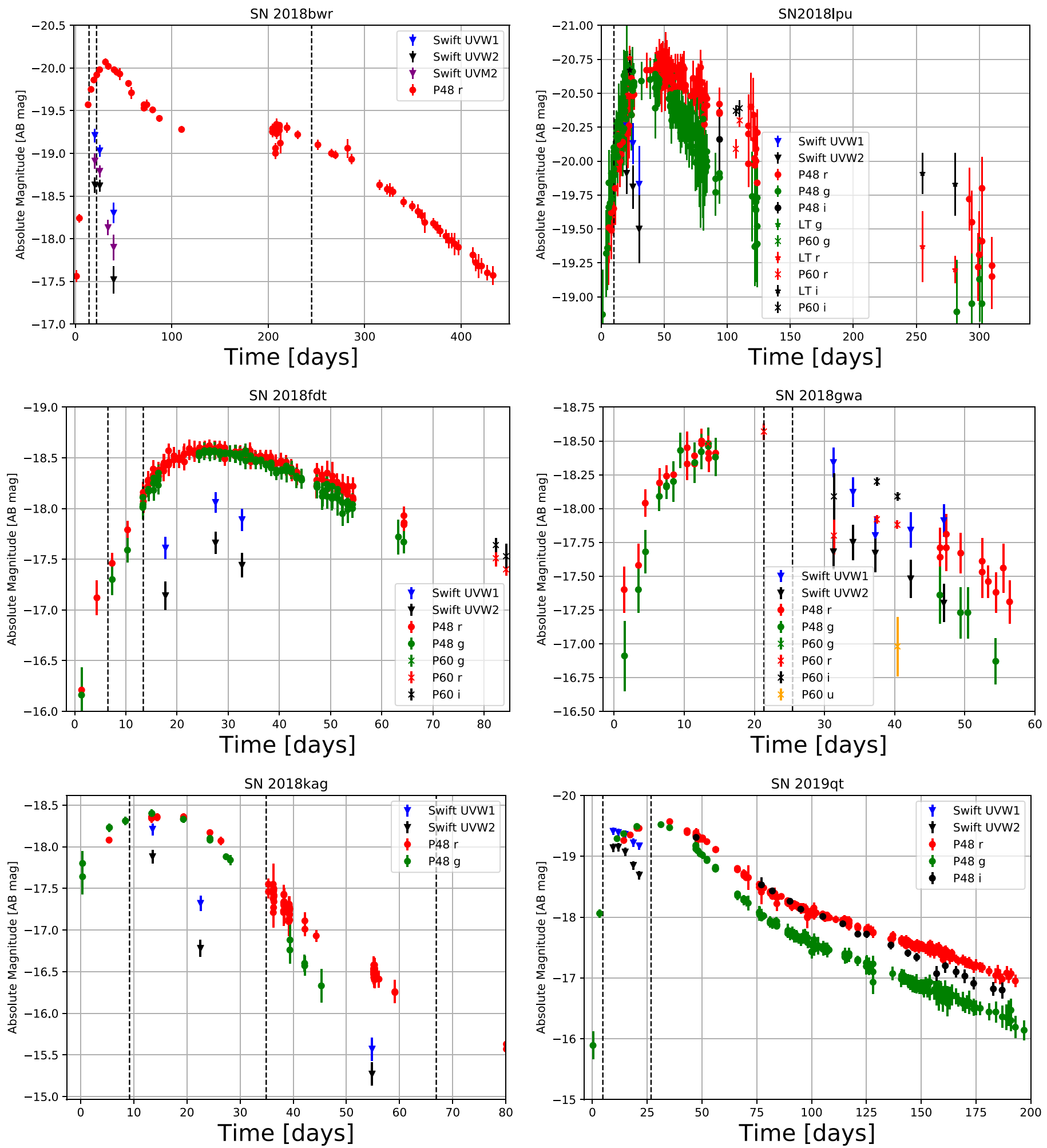

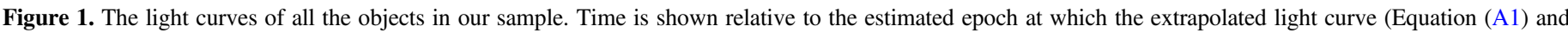

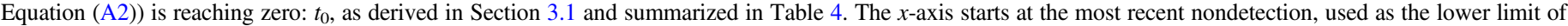

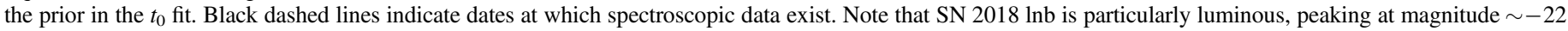

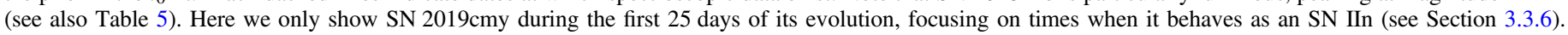

The Spectral Energy Distribution Machine (SEDm; BenAmi et al. 2012; Blagorodnova et al. 2018) spectra were automatically reduced by the IFU data reduction pipeline (Rigault et al. 2019).
The SPRAT spectra were processed by a modification of the pipeline for FrodoSpec (Barnsley et al. 2012).

The spectra taken with the Andalucia Faint Object Spectrograph and Camera (ALFOSC), mounted on the 

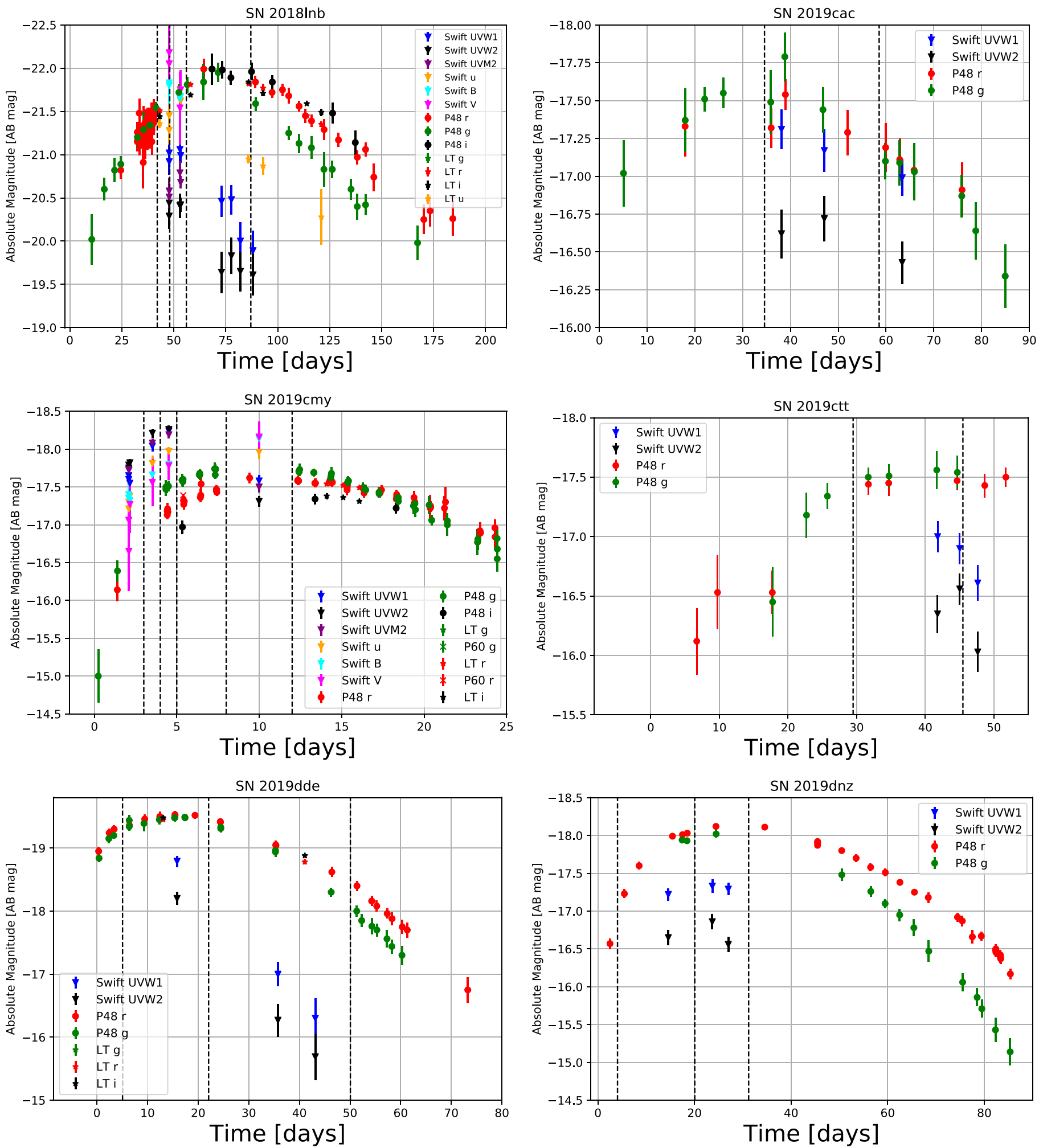

Figure 1. (Continued.)

$2.56 \mathrm{~m}$ Nordic Optical Telescope (NOT), were reduced following standard IRAF $^{25}$ procedures (Tody 1986, 1993).

25 IRAF is distributed by the National Optical Astronomy Observatories, which are operated by the Association of Universities for Research in Astronomy, Inc., under cooperative agreement with the National Science Foundation.
The spectra taken with the Auxiliary-port CAMera (ACAM), mounted on the $4.2 \mathrm{~m}$ William Herschel Telescope (WHT), were processed following standard IRAF procedures.

The data from the Double Beam Spectrograph (DBSP) on the Palomar 200 inch (P200) telescope were reduced following standard IRAF procedures of long-slit spectroscopy. The 2D 
Table 2

Photometry

\begin{tabular}{|c|c|c|c|c|c|c|c|c|}
\hline Object & $\begin{array}{l}\text { Epoch } \\
\text { (JD) }\end{array}$ & $\begin{array}{l}\text { Mag } \\
\text { (AB) }\end{array}$ & $\begin{array}{l}\text { Magerr } \\
(\mathrm{AB})\end{array}$ & $\begin{array}{c}\text { Flux } \\
\left(\mathrm{erg} \mathrm{s}^{-1}\right)\end{array}$ & $\begin{array}{l}\text { Abs. mag } \\
\text { (AB) }\end{array}$ & $\begin{array}{l}\text { Abs. magerr } \\
\text { (AB) }\end{array}$ & Filter & Instrument \\
\hline ZTF18aavskep & 2458273.8166 & 16.75 & 0.01 & $(5.267 \pm 0.049) \times 10^{-16}$ & -19.75 & 0.01 & $r$ & $\mathrm{ZTF}+\mathrm{P} 48$ \\
\hline ZTF19aadgimr & 2458502.9868 & 16.59 & 0.04 & $(1.097 \pm 0.040) \times 10^{-15}$ & -19.29 & 0.04 & $g$ & $\mathrm{ZTF}+\mathrm{P} 48$ \\
\hline ZTF19aadgimr & 2458586.8067 & 17.75 & 0.04 & $(1.366 \pm 0.050) \times 10^{-16}$ & -18.13 & 0.04 & $i$ & $\mathrm{ZTF}+\mathrm{P} 48$ \\
\hline ZTF18aavskep & 2458277.8361 & 17.87 & 0.09 & $(1.833 \pm 0.152) \times 10^{-15}$ & -18.63 & 0.09 & $U V W 2$ & Swift+UVOT \\
\hline ZTF18aavskep & 2458277.8383 & 17.58 & 0.09 & $(2.004 \pm 0.166) \times 10^{-15}$ & -18.91 & 0.09 & $U V M 2$ & Swift+UVOT \\
\hline ZTF18aavskep & 2458277.8405 & 17.29 & 0.08 & $(1.984 \pm 0.146) \times 10^{-15}$ & -19.21 & 0.08 & $U V W 1$ & Swift+UVOT \\
\hline
\end{tabular}

Note. A portion of this table is shown here for guidance regarding its form and content. Time is shown relative to the estimated epoch at which the extrapolated light curve (based on Equation (A2) and Equation (A1)) is reaching zero, as derived in Section 3.1 and shown in Table 1.

Table 3

Summary of Spectroscopic Observations

\begin{tabular}{|c|c|c|}
\hline Object & Date & Facility \\
\hline \multirow[t]{3}{*}{ SN 2018bwr } & 2018 Jun 2 & P60 + SEDM \\
\hline & 2018 Jun 10 & $\mathrm{LT}+$ SPRAT \\
\hline & 2019 Jan 19 & $\mathrm{NOT}+\mathrm{ALFOSC}$ \\
\hline SN 2018lpu & 2018 Jul 17 & P200 + DBSP [1] \\
\hline \multirow[t]{2}{*}{ SN $2018 \mathrm{fdt}$} & 2018 Aug 12 & $\mathrm{P} 60+\mathrm{SEDM}$ \\
\hline & 2018 Aug 19 & P200 + DBSP \\
\hline \multirow[t]{2}{*}{ SN 2018gwa } & 2018 Oct 6 & P60 + SEDM \\
\hline & 2018 Oct 10 & P200 + DBSP \\
\hline \multirow[t]{3}{*}{ SN 2018kag } & 2018 Dec 24 & P60 + SEDM \\
\hline & 2019 Jan 19 & $\mathrm{NOT}+\mathrm{ALFOSC}$ \\
\hline & 2019 Feb 20 & WHT + ACAM \\
\hline \multirow[t]{2}{*}{ SN 2019qt } & 2019 Jan 13 & UH88 + SNIFS * \\
\hline & 2019 Feb 04 & $\mathrm{NOT}+\mathrm{ALFOSC}$ \\
\hline \multirow[t]{7}{*}{ SN 2018lnb } & 2019 Jan 29 & $\mathrm{LT}+\mathrm{SPRAT}$ \\
\hline & $2019 \mathrm{Feb} 4$ & LCOGT $2 \mathrm{~m}+$ FLOYDS \\
\hline & $2019 \mathrm{Feb} 4$ & $\mathrm{LT}+\mathrm{SPRAT}$ \\
\hline & 2019 Feb 12 & P200 + DBSP \\
\hline & 2019 Feb 12 & P200 + DBSP \\
\hline & 2019 Mar 15 & $\mathrm{NOT}+\mathrm{ALFOSC}$ \\
\hline & 2019 Mar 15 & P60 + SEDM \\
\hline \multirow[t]{2}{*}{ SN 2019cac } & 2019 Mar 14 & $\mathrm{P} 60+\mathrm{SEDM}$ \\
\hline & 2019 Apr 07 & $\mathrm{NOT}+\mathrm{ALFOSC}$ \\
\hline \multirow[t]{7}{*}{ SN $2019 \mathrm{cmy}$} & 2019 Mar 29 & P60 + SEDM \\
\hline & 2019 Mar 30 & $\mathrm{ARC}+\mathrm{DIS}$ \\
\hline & 2019 Mar 30 & P60 + SEDM \\
\hline & 2019 Mar 30 & $\mathrm{P} 60+\mathrm{SEDM}$ \\
\hline & 2019 Mar 31 & P60 + SEDM \\
\hline & 2019 Apr 3 & Keck I + LRIS \\
\hline & 2019 Apr 7 & $\mathrm{ARC}+\mathrm{DIS}$ \\
\hline \multirow[t]{3}{*}{ SN 2019ctt } & 2019 Apr 06 & UH88 + SNIFS * \\
\hline & 2019 Apr 22 & P60+SEDM \\
\hline & 2019 Apr 24 & P200+DBSP \\
\hline \multirow[t]{3}{*}{ SN 2019dde } & 2019 Apr 14 & P60 + SEDM \\
\hline & 2019 Apr 16 & SOAR + Goodman * \\
\hline & 2019 May 1 & $\mathrm{LT}+\mathrm{SPRAT}$ \\
\hline \multirow[t]{4}{*}{ SN 2019dnz } & 2019 Apr 19 & P60 + SEDM \\
\hline & 2019 Apr 19 & $\mathrm{LT}+\mathrm{SPRAT}$ \\
\hline & 2019 Apr 30 & $\mathrm{LT}+$ SPRAT \\
\hline & 2019 May 11 & $\mathrm{LT}+$ SPRAT \\
\hline
\end{tabular}

Note. The spectra marked with a star were obtained from the TNS and kindly made available to us by Anna V Payne, Michael A. Tucker (SCAT), and Dr. Regis Cartier. [1] The 600/4000 grism and 316/7500 grating were used for the blue and red cameras, respectively, with the D55 dichroic.

images were first bias-subtracted and flat-field-corrected, then the 1D spectral spectra were extracted, wavelength-calibrated with comparison lamps, and flux-calibrated using observations of spectrophotometric standard stars observed during the same night and at approximately similar airmasses to the SN.

The spectra taken with the SuperNova Integral Field Spectrograph (SNIFS; Aldering et al. 2002; Lantz et al. 2004) were obtain from TNS with kind permission from Anna V Payne and Michael A. Tucker.

Data were taken with the FLOYDS spectrograph mounted on the $2 \mathrm{~m}$ Faulkes Telescope North, Hawaii, USA through the observing program TAU2019A-008. A 1"' 2 slit was placed on the target. The spectrum was extracted and calibrated following standard procedures using the FLOYDS data reduction pipeline ${ }^{26}$ (Valenti et al. 2014).

Data from the Dual Imaging Spectrograph (DIS) mounted on the $3.5 \mathrm{~m}$ Astrophysics Research Consortium (ARC) telescope at the Apache Point Observatory were reduced using standard procedures and calibrated to a standard star obtained the same night using the PyDIS package (Davenport et al. 2018);

Data taken with the the Keck Low-Resolution Imaging Spectrometer (LRIS) (Oke et al. 1995). The data was reduced with the LRIS automated reduction pipeline ${ }^{27}$ (Perley 2019).

\section{Analysis \\ 3.1. Epoch of Zero Flux}

In order to derive the extrapolated epoch of zero flux of all the events, we used the Photomanip ${ }^{28}$ package (released in the Appendix of this paper) to fit the $r$-band flux during the rise time (or the $g$-band flux light curve, when early $r$-band data points are not available) with an exponential function of the form

$$
f=f_{\max }\left\{1-\exp \left[\left(t_{0}-t\right) / t_{c}\right]\right\}
$$

and a power-law of the form

$$
f=a\left(t-t_{0}\right)^{n},
$$

where $t_{0}$ is the extrapolated time of zero flux, $f_{\max }$ is the maximum flux, and $t_{c}$ is the characteristic rise time of the $r$ band light curve. In each case, we chose the function giving the best fit (i.e., lowest $\chi^{2} / d o f$ ), which allowed us to estimate the epochs at which the extrapolated light curves are reaching zero, which are used throughout this paper as the reference time $t_{0}$, and are summarized in Table 4. For each SN in our sample, the table shows the band in which the fit was performed ( $g$ or $r$, depending on how constraining the data are), the prior on $t_{0}$ is

\footnotetext{
$\overline{26}$ https://github.com/svalenti/FLOYDS_pipeline

$27 \mathrm{http}: / /$ www.astro.caltech.edu/dperley/programs/lpipe.html

28 https://github.com/maayane/PhotoManip
} 

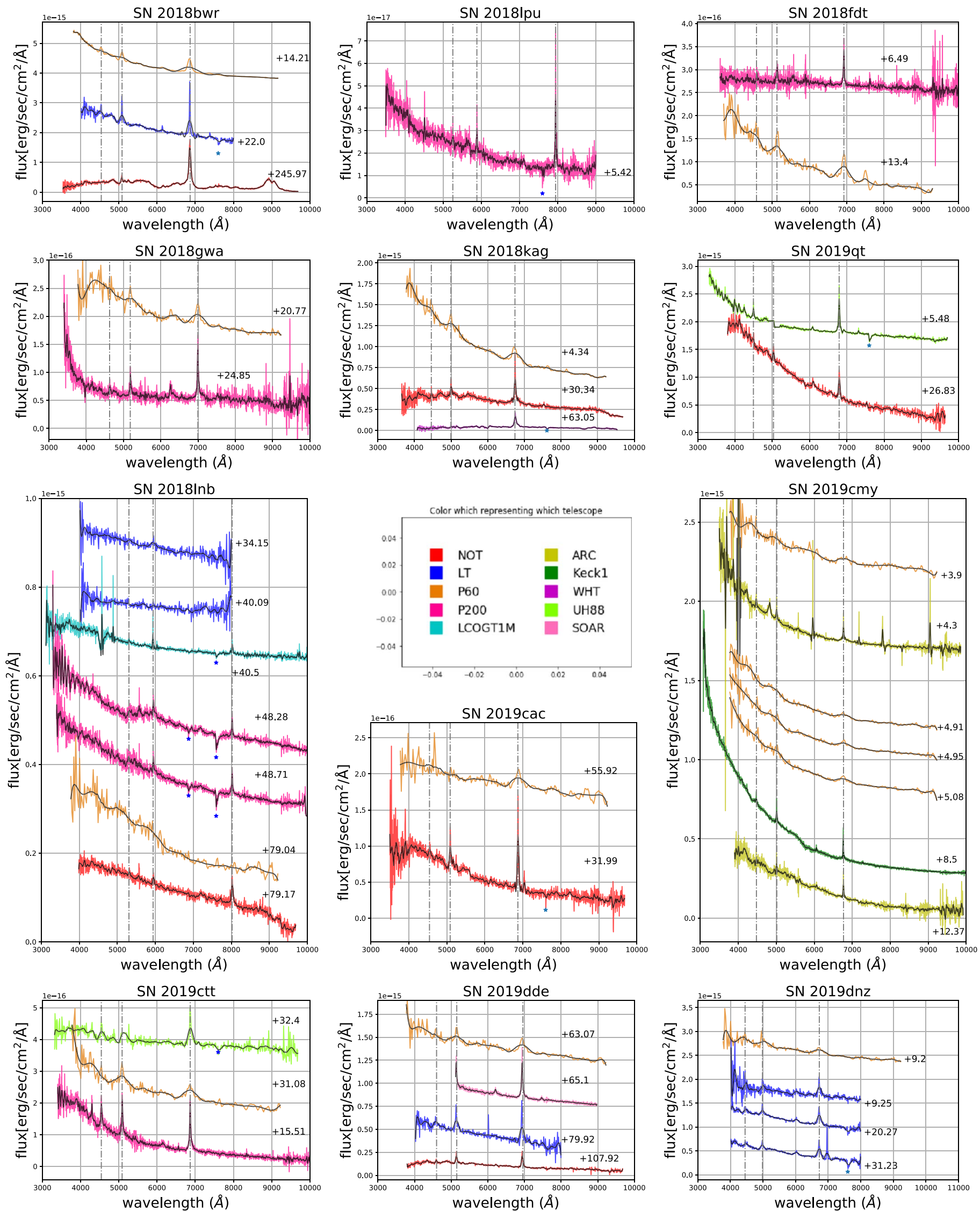

Figure 2. Optical spectra of all Type IIn SNe studied for this article. The dashed vertical lines show the Balmer series. The blue stars indicate telluric absorption. 
Table 4

Reference Times Fitting Results

\begin{tabular}{|c|c|c|c|c|c|c|}
\hline IAU Name & ZTF Name & Model & Band & $\begin{array}{l}\text { Most Recent Upper Limit } \\
(M J D)\end{array}$ & $\begin{array}{c}t_{0} \\
(M J D)\end{array}$ & $\begin{array}{c}\text { Confidence Interval } \\
(M J D)\end{array}$ \\
\hline SN 2018lpu & ZTF18abgrlpv & power law & $g$ & 58306.35 & 58306.35 & [58306.35,58306.83] \\
\hline SN 2018fdt & ZTF18abltfho & exponent & $r$ & 58334.17 & 58335.83 & {$[58335.22,58336.10]$} \\
\hline SN 2018bwr & ZTF18aavskep & exponent & $r$ & 58257.02 & 58257.03 & [58256.86,58257.13] \\
\hline SN 2018kag & ZTF18acwzyor & power law & $g$ & 58464.46 & 58467.08 & [58465.54,58467.37] \\
\hline SN 2019cac & ZTF19aaksxgp & power law & $g$ & 58521.28 & 58521.44 & [58503.48,58526.27] \\
\hline SN $2019 \mathrm{cmy}$ & ZTF19aanpcep & exponent & $g$ & 58567.48 & 58568.00 & [58567.82,58567.84] \\
\hline SN 2019ctt & ZTF19aanfqug & exponent & $r$ & 58541.30 & 58549.52 & [58546.17,58551.33] \\
\hline SN 2019dde & ZTF19aaozsuh & power law & $r$ & 58573.40 & 58581.94 & [58579.55,58582.24] \\
\hline SN 2019dnz & ZTF19aaqasrq & exponent & $r$ & 58581.50 & 58582.92 & [58582.35,58583.18] \\
\hline
\end{tabular}

Note. The "model" column specifies whether a power law (Equation (A2)) or a concave exponent (Equation (A1)) gives the best fit. The "band" column specifies the band ( $g$ or $r$ ) used for the fit, and was chosen according to the amount of data available in each band. We then report the most recent nondetection, which we use as the lower limit of our prior on $t_{0}$ (we use the most recent detection as the upper limit). For SN 2019cac, no previous nondetection exists, so our prior interval is a time interval of 100 days before the first detection. The " $t_{0}$ " column is the best-fit time at which the flux reaches zero-the time used as an estimate of the explosion epoch. The confidence interval, shown in the last column, is defined here as the tightest intervals containing $68 \%$ of the probability and including our best-fit $t_{0}$ value.

taken to be a time interval from $\sim 1$ day before the most recent pre-explosion upper limit and the first detection. Table 4 also shows the $1 \sigma$ confidence interval on $t_{0}$. The typical uncertainty on $t_{0}$ is of the order of 1 to a few days, with the exception of SN 2019cac (where no previous nondetection exists and for which we applied a broad conservative prior on $t_{0}$ ), for which it is higher than 20 days.

\subsection{Blackbody Temperature, Radius, and Bolometric Luminosity}

Taking advantage of the multiple-band photometry coverage, we used the PhotoFit ${ }^{29}$ tool (Soumagnac et al. 2019a) to derive the temperature and radius of the blackbody that best fits the photometric data at each epoch. The derived best-fit temperatures $T_{\mathrm{BB}}$ and radii $r_{\mathrm{BB}}$ are shown in Figure 3. We observe that seven objects of our sample exhibit a fast increase of the blackbody radius, a result in contrast with most previous observations. Indeed, many previously studied SNe IIn showed a constant blackbody radius (e.g., SN2010j1; Ofek et al. 2014), consistent with the continuum photosphere being located in the unshocked optically thick CSM. In some cases a blackbody radius stalling after a short increase (e.g., 2005kj, 2006bo, 2008fq, 2006qq, Taddia et al. 2013; 2006tf, Smith et al. 2008) or even a shrinking blackbody radius (e.g., SN2005ip; SN2006jd, Taddia et al. 2013) were observed. Such observations were explained by the possible presence of clumps in the CSM that may expose underlying layers (Smith et al. 2008). PTF 12glz was not the only case were a fast increase of the blackbody radius was observed: three of the SNe IIn observed-in the UV -by de la Rosa et al. (2016) showed blackbody radii growing at comparable rates. This could be due to the fact that UV observations provide a better handle on the blackbody spectrum shape than visible light alone, suggesting that a fast increase of the blackbody radius of SNe IIn may be more common than suggested by visible-light surveys of these objects.

We further discuss and exploit the $r_{\mathrm{BB}}$ measurement in Section 4 .

${ }^{29}$ https://github.com/maayane/PhotoFit
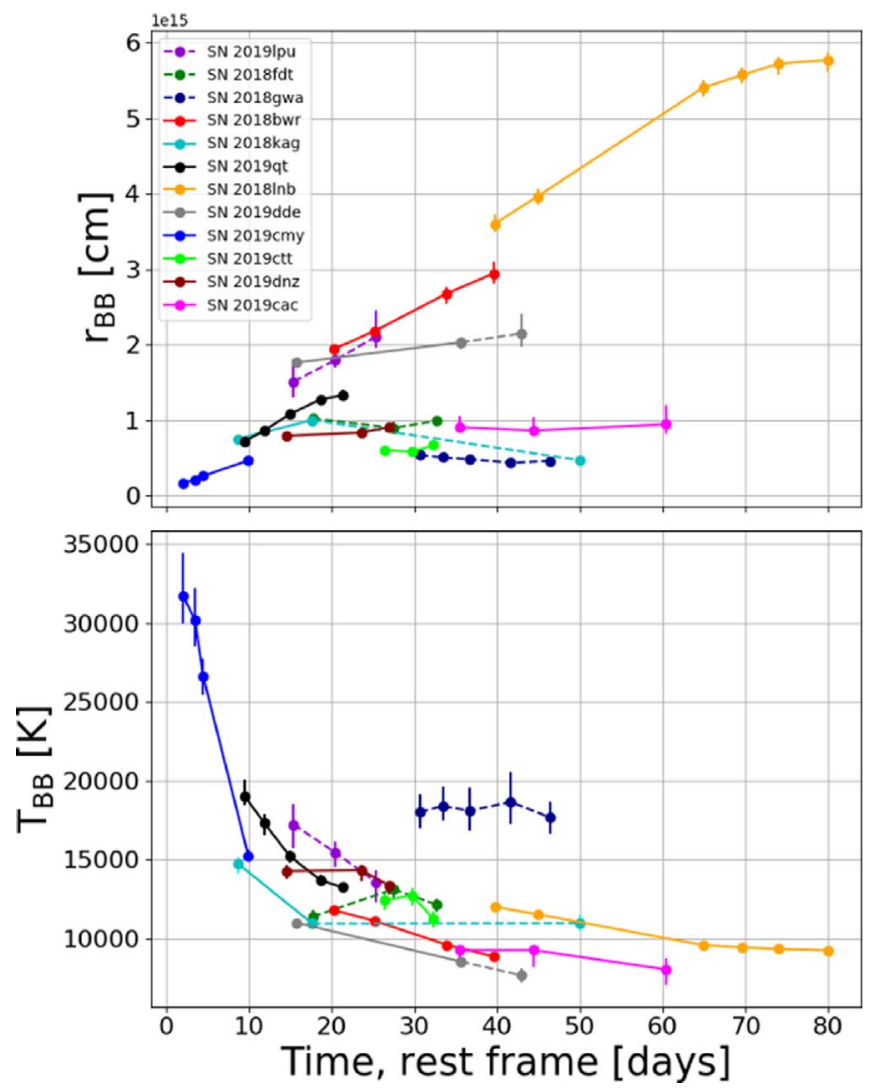

Figure 3. Evolution in time of: (1) the radius (upper panel), (2) the temperature (lower panel) of a blackbody with the same radiation as each of the $12 \mathrm{SNe}$ in our sample. The points were obtained by fitting a blackbody spectrum to the observed photometry, after interpolating the various data sets to obtain data coverage of coinciding epochs. The errors were obtained with Monte Carlo Markov chain simulations. The dashed lines correspond to objects for which no late spectra were obtained in order to confirm that the CSM is optically thick. They should be taken cautiously.

Based on the measurement of $r_{\mathrm{BB}}$ and $T_{\mathrm{BB}}$, we were able to derive the luminosity $L_{\mathrm{BB}}=4 \pi r_{\mathrm{BB}}^{2} \sigma T_{\mathrm{BB}}^{4}$ of the blackbody fits, shown in Figure 4. 


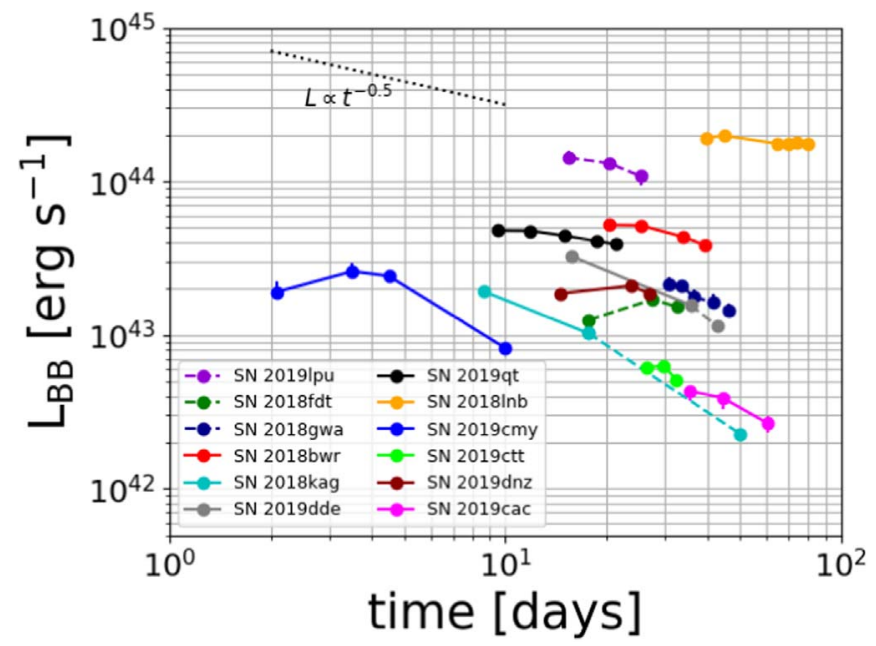

Figure 4. The evolution in time of the bolometric luminosity of a blackbody with the same radiation as each of the $12 \mathrm{SNe}$ in our sample. The dashed lines correspond to objects for which no late spectra were obtained in order to confirm that the CSM is optically thick. They should be taken cautiously. The dotted line shows the $t^{-0.5}$ slope (see, e.g., Ofek et al. 2014).

\subsection{Spectroscopy}

In this section, we only report the spectroscopic information that allow us to assess which photometric data are usable for our analysis of the CSM geometry. Indeed, the asphericity criterion proposed by Soumagnac et al. (2019b) is only applicable at times when the CSM surrounding the explosion is optically thick. To verify this, we require that the spectrum be dominated by a blackbody continuum with no high-velocity $\left(2000 \mathrm{~km} \mathrm{~s}^{-1}\right)$ absorption and emission lines.

We can only include in our analysis multiple-band photometry that was collected before, or close to, the observation of a spectrum showing no evidence for highvelocity material. Unfortunately, we were unable to secure such spectroscopy for the SNe IIn SN 2018lpu, SN 2018fdt, and SN 2018gwa, for which no spectra were taken after or close to the last Swift data point.

\subsubsection{SN $2018 b w r$}

The first two spectra show $\mathrm{H}_{\alpha}, \mathrm{H}_{\beta}$, and $\mathrm{H}_{\gamma}$ emission lines. In the last spectrum, we see prominent broad Ca II emission, blended with the O I $\lambda 8446 \AA$ feature. The numerous Fe lines are blended, exhibiting a pseudo-continuum around $\sim 5500 \AA$. Such a pseudo-continuum is also seen, e.g., in PTF $12 \mathrm{glz}$ (Soumagnac et al. 2019b) and in SN 2005cl (Kiewe et al. 2012). We conclude from this that the spectra are dominated by interaction out to late times, and we can use all of the UV photometry for our analysis.

\subsubsection{SN $2018 \mathrm{kag}$}

The first spectrum shows a blue continuum with Balmer emissions lines. The Balmer lines remain discernible at +30.3 $\mathrm{d}$ and the continuum becomes flat. At $+63.10 \mathrm{~d}$, highervelocity absorption and emission lines have appeared in the spectrum, hinting that the CSM may not be optically thick anymore. As a result, only the UV photometry taken between the first two spectra is usable for our analysis of the CSM geometry.

\subsubsection{SN $2019 q t$}

Distinct narrow $\mathrm{H}_{\alpha}$ and $\mathrm{H}_{\beta}$ emission lines are visible in both spectra. $\mathrm{H}_{\gamma}$ emission is also visible, especially in the earlier spectrum. Since all the UV photometry was taken between the epochs of these two spectra, all of it is usable for our analysis.

\subsubsection{SN $2018 \ln b$}

Narrow Balmer emission is visible in all spectra except for the first two spectra, in which the $\mathrm{H} \alpha$ component falls outside the spectral range of SPRAT/LT, and the SEDm/P60 spectrum, which has low signal-to-noise. All of the UV photometric data are usable for our analysis.

\subsubsection{SN 2019cac}

In spite of the low resolution of the first spectrum, $\mathrm{H}_{\alpha}$ emission is visible at +31.9 days. Strong emission lines of $\mathrm{H}_{\alpha}$, $\mathrm{H}_{\beta}$, and $\mathrm{H}_{\gamma}$ can be observed at +55.9 days. Although the last UV data point was taken after the second spectrum, we consider their epochs to be close enough that all of the UV data can be used for our analysis.

\subsection{6. $S N 2019 \mathrm{cmy}$}

The limit between flash-spectroscopy events and Type IIn $\mathrm{SNe}$ can be blurry, when the Balmer lines persist for weeks or a few months.

In the case of SN 2019cmy, prominent narrow Balmer emissions lines are visible at +4.9 days, with the characteristic broad wings of the $\mathrm{H} \alpha$ line, interpreted as the signature of electron scattering, clearly visible. Strong high-ionization emission lines of He II $\lambda 4686 \AA$ only persist at +4.9 days. An excess on the blue side of the He II $\lambda 4686 \AA$ coincides with the high-ionized C III $\lambda 4650 \AA$. However, by +8.5 days, the C III $\lambda 4650 \AA$ and He II $\lambda 4686 \AA$ lines have completely disappeared, consistent with flash-ionized emissions. The Balmer lines decrease in strength with time: the $\mathrm{H}_{\gamma} \lambda 4341 \AA$ and $\mathrm{H}_{\delta} \lambda 4102 \AA$ are marginally detected at +8.5 days and have disappeared by day +12.4 . A spectrum taken two months after first light (and not shown in this paper) exhibits the features of a "normal" Type II SN, without any particular signature of CSM interaction.

Our geometrical analysis, which probes the shape of the CSM rather than its amount or the physical ways by which it was ejected, should still hold at early times. All the UV photometry is usable for our analysis.

\subsubsection{SN $2019 c t t$}

Narrow Balmer lines $\left(\mathrm{H}_{\alpha}, \mathrm{H}_{\beta}, \mathrm{H}_{\gamma}\right)$ are visible in all three spectra. The $\mathrm{H}_{\delta}$ line is also visible in the higher-resolution spectrum at +32.4 days. All the UV photometry is usable for our analysis.

\subsubsection{SN 2019dde}

The first spectrum, taken at +63.07 days with the SEDm/ P60 shows narrow Balmer lines $\left(\mathrm{H}_{\alpha}, \mathrm{H}_{\beta}, \mathrm{H}_{\gamma}, \mathrm{H}_{\delta}, \mathrm{H}_{\epsilon}\right)$. The three later spectra at +65.10 days, +79.92 days, and +107.92 days show narrow $\mathrm{H}_{\alpha}$ and $\mathrm{H}_{\beta}$ emission lines,

In the last spectrum, a narrow He $\lambda 5876 \AA$ emission line is visible. Although the Balmer series is strongly dominated by narrow emission, the broad absorption at $5000-10,000 \mathrm{~km} \mathrm{~s}^{-1}$ 
suggests that the ejecta have become visible, and the CSM is not completely optically thick anymore.

To account for this, we only use the first two UV epochs for our analysis.

\subsection{9. $S N 2019 d n z$}

Narrow Balmer lines $\left(\mathrm{H}_{\alpha}, \mathrm{H}_{\beta}, \mathrm{H}_{\gamma}\right)$ are visible in all three spectra. In addition, $\mathrm{H}_{\delta}, \mathrm{H}_{\epsilon}$ emission lines can be seen in the last spectrum. All the UV photometry can be used for our analysis.

\subsubsection{Events with Missing Final Spectra}

For three objects in our sample, we were unable to collect a spectrum showing no evidence for high-velocity material close to or after the last UV photometry epoch. For SN 2018 lpu, one spectrum was taken, where strong and narrow Balmer lines can be seen. Other interesting features include narrow emission of He II ( $\lambda 3203 \AA, \lambda 4686 \AA)$, [O II] $\lambda 3727 \AA$, and [O III] $\lambda 5007 \AA$. For both SN 2018fdt and SN 2018gwa, two spectra were obtained before any Swift photometry was taken. Both show prominent narrow Balmer emissions lines.

\section{Fraction of SNe IIn Showing Evidence for Aspherical CSM}

In all that follows, we assume that the criterion from Soumagnac et al. (2019b), i.e., a fast increase of the blackbody effective radius (if observed at times when the CSM surrounding the explosion is still optically thick), is an indication for asphericity. We note that asphericity could manifest in other ways, and that this approach does not allow us to exclude or constrain more complicated geometries.

\subsection{Application of the Asphericity Criterion from Soumagnac et al. (2019b)}

Assessing whether the blackbody radius $r_{\mathrm{BB}}$, shown in Figure 3, is growing or not, is a hypothesis testing problem, i.e., we need to select between two models the one that best explains the data. Our model is a power-law function of the form $R=R_{0}\left(\frac{t}{t_{0}}\right)^{n}$, where the null hypothesis is that $n=0$ and the alternative hypothesis is that $n \neq 0$. Since these models are nested, we can apply a likelihood-ratio test (or chi-squared difference test) to discriminate between them. In Figure 5, we show the $\chi^{2}$ difference between the two models derived for all objects. For six out of nine objects, $\Delta \chi^{2}>4$ i.e., the chisquared difference indicates that the increasing radius is more likely than the constant radius at a $2 \sigma$ level. Therefore, $66 \%$ of the SNe in our sample (taking into account only the $\mathrm{SNe}$ to which our analysis is applicable) show evidence for aspherical CSM.

\subsection{Do Brighter SNe IIn Have More Aspherical CSM? Correction for Potential Selection Effects}

In Figure 6, we show the distribution of absolute magnitudes of the SNe IIn in our sample. The overall distribution (in blue) is comparable to a previously published absolute luminosity distribution for SNe IIn (see, e.g., Figure 17 in Richardson et al. 2014). However, the SNe showing no evidence for a rising $r_{\mathrm{BB}}$ are on the faint end of the distribution. This trend is also visible in Figure 7, where we show the lower limit on the

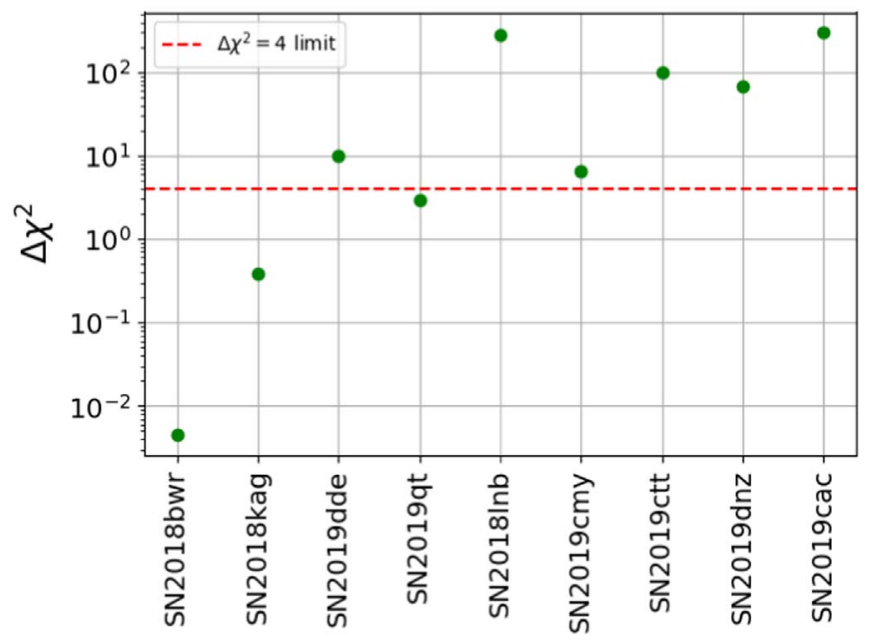

Figure 5. Result of the likelihood-ratio test (or chi-squared difference test), when modeling the evolution of $r_{\mathrm{BB}}$ with a power law and with a flat function. The red dashed line shows the $\Delta \chi^{2}=4$ (i.e., $2 \sigma$ ) limit for one degree of freedom difference: objects with a $\Delta \chi^{2}$ limit above this line are better modeled by a non-zero power law (and hence show evidence for aspherical CSM), whereas objects below this line are better modeled by a flat line (i.e., show no evidence for aspherical CSM). Applying the criterion from Soumagnac et al. (2019b), six out of nine SNe IIn in our sample show evidence for aspherical $\mathrm{CSM}$. The SNe are ordered by their maximum measured bolometric luminosity (left to right)

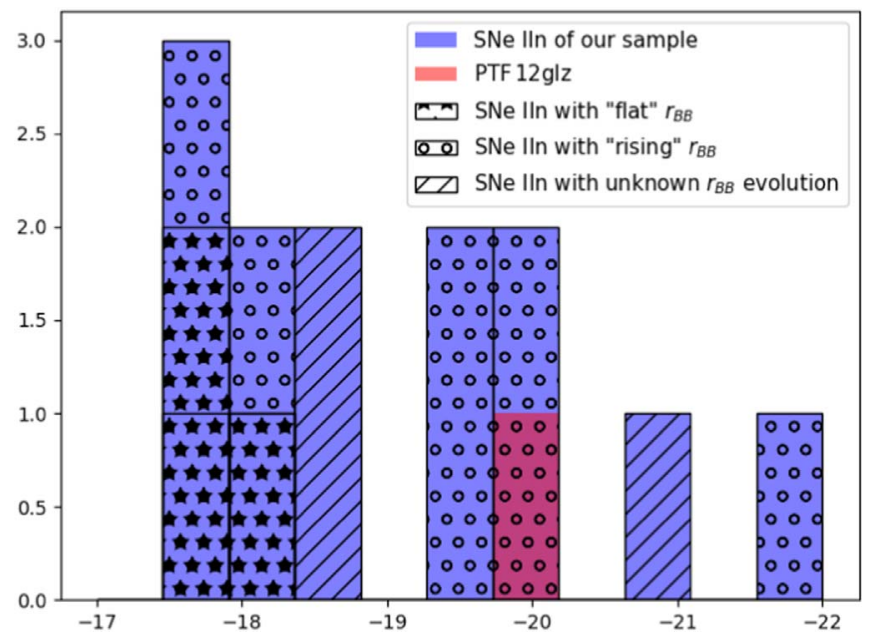

Figure 6. Absolute magnitude of the $12 \mathrm{SNe}$ IIn of our sample and PTF 12glz. The blue histograms correspond to the entire sample and the red square corresponds to PTF 12glz. The star-patterned histograms correspond to the $\mathrm{SNe}$ IIn whose radius is better modeled by a flat function than by a power law (i.e., showing no evidence for aspherical CSM). These objects are at the faint end of the distribution, an effect we need to correct for in the calculation of their probability of occurring (see Section 4.2). The circle-patterned histograms correspond to the SNe IIn whose radius is better modeled by a power law (i.e., showing evidence for aspherical CSM). The line-patterned histograms correspond to the SNe IIn discussed in Section 3.3.10, i.e., for which no late spectrum was collected and to which our analysis of the CSM geometry does not apply.

bolometric luminosity of all the SNe IIn in our sample (also reported in Table 5), as a function of (1) the index of the power law that best fits $r_{\mathrm{BB}}$ and (2) the $\chi^{2}$ difference between the two models derived for all objects (also reported in Table 5; see Section 4.1).

The objects of our sample that are intrinsically brighter appear to show evidence for an increasing blackbody radiuswhich we interpret as an indication for aspherical CSM- 

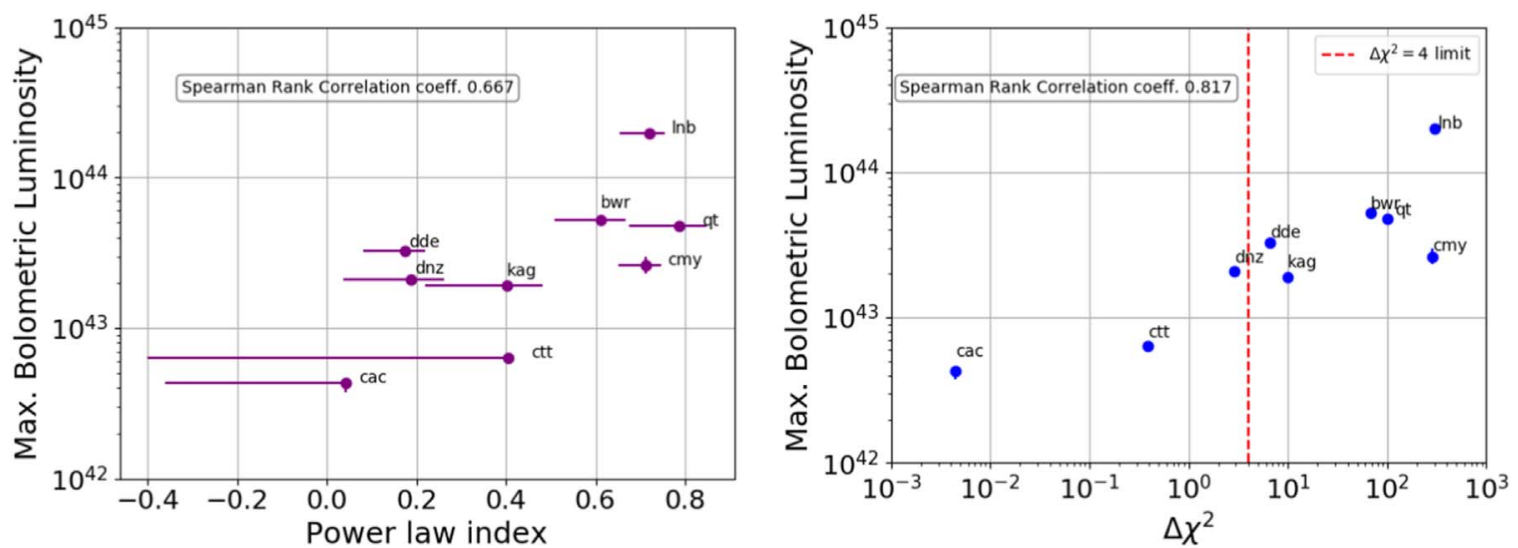

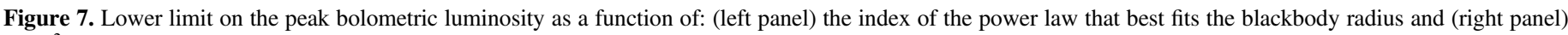

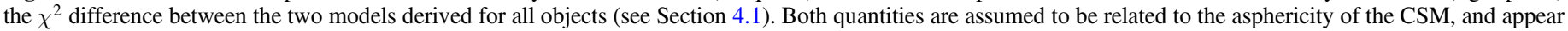
to be correlated with the peak bolometric luminosity.

Table 5

Bolometric Luminosity and Asphericity of the CSM

\begin{tabular}{lcc}
\hline \hline IAU Name & $\begin{array}{c}\text { lower limit on peak } L_{\mathrm{BB}} \\
\left(\mathrm{erg} \mathrm{s}^{-1}\right)\end{array}$ & $\Delta \chi^{2}$ \\
\hline SN2018bwr & $5.22 \times 10^{43}$ & 68.6 \\
SN2018kag & $1.92 \times 10^{43}$ & 9.8 \\
SN2019dde & $3.26 \times 10^{43}$ & 6.5 \\
SN2019qt & $4.80 \times 10^{43}$ & 99.3 \\
SN2018lnb & $19.87 \times 10^{44}$ & 302.0 \\
SN2019cmy & $2.60 \times 10^{43}$ & 284.6 \\
SN2019ctt & $6.32 \times 10^{42}$ & 0.4 \\
SN2019dnz & $2.10 \times 10^{43}$ & 2.9 \\
SN2019cac & $4.33 \times 10^{42}$ & 0.0 \\
\hline
\end{tabular}

Note. Lower limit on the peak bolometric luminosity $L_{\mathrm{BB}}$ and $\chi^{2}$ difference between a power-law model with $n \neq 0$ and $n=0$.

whereas fainter objects tend not to show such a feature. The Spearman rank correlation between the power-law index and the lower limit on the bolometric luminosity is 0.67 , and 0.82 between the lower limit on the bolometric luminosity and $\Delta \chi^{2}$. The false alarms probabilities are 0.03 and 0.005 , respectively (the false alarm probability were estimated using bootstrap simulations implemented in Ofek 2014).

There are several possible explanations to this correlation. It could be the result of either some selection bias or some physical reasons (or a combination of both). Among the possible physical reasons are the following: (i) more massive, or alternatively more energetic explosions, tend to occur in aspherical CSM; (ii) a geometrical effect, related to the viewing angle, could also be playing a role. Indeed, if one thinks about a slab of CSM (for simplicity), the increase of the blackbody radius, which we used in this paper as a criterion for asphericity, is most patent when the explosion is observed perpendicularly to the long axis of the slab. The brighter events of our sample could happen to be observed from this direction, while the fainter events could be observed from the short-axis direction, preventing us from detecting the asphericity of their CSM using our criterion. We plan to explore this effect in future work.

The observed correlation could also be due to some selection effects. If we assume that both classes of SNe IIn obey the same luminosity - and volume - distribution, the SNe showing no evidence for a rising $r_{\mathrm{BB}}$ appear to be underrepresented in our sample, a fact that needs to be corrected for in the final probability calculation. (A full relative rate calculation, taking into account a broader variety of selection effects, e.g., due to the cadence, the varying limiting magnitude of each image or the extinction at the location of the $\mathrm{SN}$, is beyond the scope of this paper). Here, we simply estimate the relative probability $P_{i}$ of finding the $i$ th $\mathrm{SN}$ IIn of our sample $\left(\mathrm{SN}_{i}\right)$ as

$$
p_{i}=\frac{\frac{1}{V_{\max , i}}}{\sum_{j=1}^{9} \frac{1}{V_{\max , j}}},
$$

where $V_{\max , i}$ is the maximum volume to which $\mathrm{SN}_{i}$ can be observed, under the assumption of a constant limiting magnitude for the survey in the $r, m_{\text {lim }}=20.5$. In case both classes of objects obey the same luminosity distribution, the corrected fraction of SNe IIn exhibiting a rising $r_{\mathrm{BB}}$ is $35 \%$.

To conclude, depending on the assumption we make on the luminosity distribution of both classes of objects, the fraction of SNe IIn showing an increasing radius, deduced from our sample, could be $35 \%$, or as high as $66 \%$. As this is a sufficient but not necessary condition for the surrounding CSM to be aspherical, these numbers are a lower limit on the fraction of SNe IIn exploding in aspherical CSM.

\section{Conclusions}

We presented the first planned ultraviolet (UV) survey of the early evolution of type IIn supernovae (SNe IIn). Our sample consists of $12 \mathrm{SNe}$ IIn discovered and observed with the Zwicky Transient Facility (ZTF) and followed up in the UV by the Neil Gehrels Swift Observatory. All SNe were also spectroscopically followed up: we present and release the spectroscopic data we collected.

The UV observations presented in this paper could help shed light on various aspects of the physical picture governing these events. For example, they may be used to better understand the explosion mechanism and the CSM properties (e.g., Ofek et al. 2013a), since the collisionless shock propagating in the CSM after the shock breakout (Ofek et al. 2010) is predicted to radiate most in the $\mathrm{UV}$ and $\mathrm{X}$-rays.

Observations of SNe IIn at UV wavelengths provide more insights into the bolometric luminosity, blackbody radius, and blackbody temperature than visible-light observations alone. This may be a reason why the fast rising blackbody radius- 
which we observe for 7 objects out of the 12 of our samplewas only observed in the past in works using UV observations of SNe IIn (de la Rosa et al. 2016; Soumagnac et al. 2019b). This result is in contrast with most previous observations using visible-light observations alone, of either a constant, slowly rising (and then stalling), or even a shrinking blackbody radius.

Assuming that a rising blackbody radius is an indication of asphericity, we used the UV observations to address the following question: "what fraction of SNe IIn explode in aspherical CSM?" Indeed, although observations of SNe IIn are usually analyzed within the framework of spherically symmetric models of CSM, resolved images of stars undergoing considerable mass loss as well as polarimetry observations suggest that asphericity is common, and should be taken into account for realistic modeling of these events. Constraining the geometrical distribution of the CSM surrounding the explosion is key to understanding the mass-loss processes occurring before the explosion and the nature of the yet-to-be determined progenitors of SNe IIn. Indeed, the presence of aspherical CSM around the progenitor is hard to explain by a simple wind, and requires other scenarios, such as episodic emission, rapid stellar rotation, or binarity.

We applied the criterion for asphericity introduced by Soumagnac et al. (2019b), stating that a fast increase of the blackbody effective radius, if observed at times when the CSM surrounding the explosion is still optically thick, may be interpreted as an indication that the CSM is aspherical. We find that two-thirds of the $\mathrm{SNe}$ in our sample show evidence for aspherical CSM. We also find that higher-luminosity objects tend to show evidence for aspherical CSM. This correlation could be due to physical reasons or to some selection bias. If we assume that both classes of SNe In obey the same luminosity - and volume-distribution, the fraction of SNe showing evidence for a rising blackbody radius needs to be corrected. Depending on the assumption we make on the luminosity distribution of both classes of objects, the lower limit deduced from our sample on the fraction of SNe IIn showing evidence for aspherical CSM could be $35 \%$, or as high as $66 \%$. This result suggests that asphericity of the CSM surrounding $\mathrm{SNe}$ IIn is common-consistent with data from resolved images of stars undergoing considerable mass loss. It also suggests that asphericity should be taken into account for more realistic modeling of these events.

As future wide-field transient surveys and the ULTRASAT UV satellite mission (Sagiv et al. 2014) are deployed, more UV observations of interracting $\mathrm{SNe}$ will be collected, allowing us to build upon this survey and to refine the lower limit derived in this paper.

M.T.S. thanks Charlotte Ward and Eli Waxman for useful discussions.

This work is based on observations obtained with the Samuel Oschin Telescope 48 inch and the 60 inch Telescope at the Palomar Observatory as part of the Zwicky Transient Facility project. ZTF is supported by the National Science Foundation under grant No. AST-1440341 and a collaboration including Caltech, IPAC, the Weizmann Institute for Science, the Oskar Klein Center at Stockholm University, the University of Maryland, the University of Washington, Deutsches Elektronen-Synchrotron and Humboldt University, Los Alamos National Laboratories, the TANGO Consortium of Taiwan, the University of Wisconsin at Milwaukee, and Lawrence
Berkeley National Laboratories. Operations are conducted by COO, IPAC, and UW.

We acknowledge the use of public data from the Swift data archive.

SED Machine is based upon work supported by the National Science Foundation under grant No. 1106171.

This paper uses observations made with the Nordic Optical Telescope, operated by the Nordic Optical Telescope Scientific Association at the Observatorio del Roque de los Muchachos, La Palma, Spain, of the Instituto de Astrofisica de Canarias.

Some of the data we present were obtained with ALFOSC, which is provided by the Instituto de Astrofisica de Andalucia (IAA) under a joint agreement with the University of Copenhagen and NOTSA. The Liverpool Telescope, located on the island of La Palma in the Spanish Observatorio del Roque de los Muchachos of the Instituto de Astrofisica de Canarias, is operated by Liverpool John Moores University with financial support from the UK Science and Technology Facilities Council. The ACAM spectroscopy was obtained as part of OPT $/ 2018 \mathrm{~B} / 011$.

We would like to thank occasional observers on the UW APO ZTF follow-up team, including: Eric Bellm, Zach Golkhou, James Davenport, Daniela Huppenkothen, Dino Bekte $^{\circ}$ evifá Gwendolyn Eadie, and Bryce T. Bolin. M.L.G. acknowledges support from the DIRAC Institute in the Department of Astronomy at the University of Washington. The DIRAC Institute is supported through generous gifts from the Charles and Lisa Simonyi Fund for Arts and Sciences, and the Washington Research Foundation.

This work was supported by the GROWTH project funded by the National Science Foundation under grant No. 1545949.

A.G.-Y. is supported by the EU via ERC grant No. 725161, the Quantum Universe I-Core program, the ISF, the BSF Transformative program, IMOS via ISA, and by a Kimmel award. This research has made use of data and/or software provided by the High Energy Astrophysics Science Archive Research Center (HEASARC), which is a service of the Astrophysics Science Division at NASA/GSFC.

M.T.S. acknowledges support by a grant from IMOS/ISA, the Benoziyo center for Astrophysics at the Weizmann Institute of Science. This work was in part supported by the Scientific Discovery through Advanced Computing (SciDAC) program funded by U.S. Department of Energy Office of Advanced Scientific Computing Research and the Office of High Energy Physics. This research used resources of the National Energy Research Scientific Computing Center (NERSC), a U.S. Department of Energy Office of Science User Facility operated under Contract No. DE-AC02-05CH11231.

E.O.O is grateful for the support by grants from the Israel Science Foundation, Minerva, Israeli Ministry of Science, the US-Israel Binational Science Foundation, the Weizmann Institute and the I-CORE Program of the Planning and Budgeting Committee and the Israel Science Foundation.

C.F gratefully acknowledges support of his research by the Heising-Simons Foundation (\#2018-0907).

A.A.M. is funded by the Large Synoptic Survey Telescope Corporation, the Brinson Foundation, and the Moore Foundation in support of the LSSTC Data Science Fellowship Program; he also receives support as a CIERA Fellow by the CIERA Postdoctoral Fellowship Program (Center for Interdisciplinary Exploration and Research in Astrophysics, Northwestern University). 
M.R. has received funding from the European Research Council (ERC) under the European Union's Horizon 2020 research and innovation program (grant agreement No. 759194 - USNAC).

Software: ZTF pipeline (Masci et al. 2019), ZOGY (Zackay et al. 2016), HEAs oft (v6.26; Blackburn 1995; Blackburn et al. 1999; HEASARC 2014), IRAF (Tody 1986, 1993), Photomanip, PyDIS (Davenport et al. 2018), LRIS pipeline (Perley 2019), PhotoFit (Soumagnac et al. 2019a), Astropy (Astropy Collaboration et al. 2013, 2018), Matplotlib (Hunter 2007), Scipy (Virtanen et al. 2020).

\section{Appendix \\ Release of the Photomanip code}

The PhotoManip tool, used to calculate the reference time for all the light curves and figures in this paper, is made available at https://github.com/maayane/PhotoManip.

The reference time is calculated as the epochs at which the extrapolated light curve is reaching zero. PhotoManip fits either the $r$-band or the $g$-band flux during the rise time with an exponential function of the form

$$
f=f_{\max }\left\{1-\exp \left[\left(t_{0}-t\right) / t_{c}\right]\right\},
$$

and a power-law of the form

$$
f=a\left(t-t_{0}\right)^{n},
$$

(where $t_{0}$ is the time of zero flux, $f_{\max }$ is the maximum flux, $t_{c}$ is the characteristic rise time of the bolometric light curve). The fit uses the MCMC algorithm emcee (Foreman-Mackey et al. 2013).

\section{ORCID iDs}

Maayane T. Soumagnac (iD https://orcid.org/0000-00016753-1488

Eran O. Ofek (iD https://orcid.org/0000-0002-6786-8774 Avishay Gal-yam (iD https://orcid.org/0000-0002-3653-5598 Peter Nugent (iD https://orcid.org/0000-0002-3389-0586 S. Bradley Cenko (i) https://orcid.org/0000-0003-1673-970X Jesper Sollerman (iD https://orcid.org/0000-0003-1546-6615 Daniel A. Perley (iD https://orcid.org/0000-0001-8472-1996 Igor Andreoni (iD https://orcid.org/0000-0002-8977-1498 Kevin B. Burdge (iD https://orcid.org/0000-0002-7226-836X Rachel J. Bruch (1) https://orcid.org/0000-0001-8208-2473 Kishalay De (D) https://orcid.org/0000-0002-8989-0542 Christoffer Fremling (iD https://orcid.org/0000-00024223-103X

Melissa L. Graham (D) https://orcid.org/0000-0002-9154-3136 Matthew J. Hankins (iD https://orcid.org/0000-0001-9315-8437 Nora Linn Strotjohann (iD https://orcid.org/0000-00024667-6730

James D. Neill (1D https://orcid.org/0000-0002-0466-1119 Steve Schulze (i) https://orcid.org/0000-0001-6797-1889 David L. Shupe (ib) https://orcid.org/0000-0003-4401-0430 Kirsty Taggart (iD https://orcid.org/0000-0002-5748-4558 Leonardo Tartaglia (i) https://orcid.org/0000-0003-3433-1492 Lin Yan (D) https://orcid.org/0000-0003-1710-9339 Yuhan Yao (iD) https://orcid.org/0000-0001-6747-8509 Eric C. Bellm (1) https://orcid.org/0000-0001-8018-5348 Richard Dekany (i) https://orcid.org/0000-0002-5884-7867 Dmitry A. Duev (D) https://orcid.org/0000-0001-5060-8733
Sara Frederick (iD https://orcid.org/0000-0001-9676-730X Matthew J. Graham (iD https://orcid.org/0000-0002-3168-0139 Russ R. Laher (i) https://orcid.org/0000-0003-2451-5482 Frank J. Masci (i) https://orcid.org/0000-0002-8532-9395 Mansi M. Kasliwal (i) https://orcid.org/0000-0002-5619-4938 Marek Kowalski (iD https://orcid.org/0000-0001-8594-8666 Thomas Kupfer (iD https://orcid.org/0000-0002-6540-1484 Adam A. Miller (iD https://orcid.org/0000-0001-9515-478X Mickael Rigault (ib https://orcid.org/0000-0002-8121-2560

\section{References}

Aldering, G., Adam, G., Antilogus, P., et al. 2002, Proc. SPIE, 4836, 61 Aretxaga, I., Benetti, S., Terlevich, R. J., et al. 1999, MNRAS, 309, 343 Astropy Collaboration, Price-Whelan, A. M., Sipőcz, B. M., et al. 2018, AJ, 156,123

Astropy Collaboration, Robitaille, T. P., Tollerud, E. J., et al. 2013, A\&A, 558, A33

Barnsley, R. M., Smith, R. J., \& Steele, I. A. 2012, AN, 333, 101

Bellm, E. C., Kulkarni, S. R., Graham, M. J., et al. 2019, PASP, 131, 018002 Ben-Ami, S., Konidaris, N., Quimby, R., et al. 2012, Proc. SPIE, 8446, 844686 Bilinski, C., Smith, N., Williams, G. G., et al. 2018, MNRAS, 475, 1104

Blackburn, J. K. 1995, in ASP Conf. Ser. 77, Astronomical Data Analysis Software and Systems IV, ed. R. A. Shaw, H. E. Payne, \& J. J. E. Hayes (San Francisco, CA: ASP), 367

Blackburn, J. K., Shaw, R. A., Payne, H. E., Hayes, J. J. E., \& HEASARC 1999, FTOOLS: A general package of software to manipulate FITS files, Astrophysics Source Code Library, ascl:9912.002

Blagorodnova, N., Neill, J. D., Walters, R., et al. 2018, PASP, 130, 035003

Breeveld, A. A., Landsman, W., Holland, S. T., et al. 2011, in AIP Conf. Ser. 1358, Gamma-ray Burst 2010, ed. J. E. McEnery, J. L. Racusin, \& N. Gehrels (Melville, NY: AIP), 373

Cardelli, J. A., Clayton, G. C., \& Mathis, J. S. 1989, ApJ, 345, 245

Cartier, R., Briceno, C., Gomez, D., Espinoza, J., \& Estay, O. 2019, ATel, 12671

Cenko, S. B., Fox, D. B., Moon, D.-S., et al. 2006, PASP, 118, 1396

Chevalier, R. A., \& Irwin, C. M. 2012, ApJL, 747, L17

Chugai, N. N., \& Danziger, I. J. 1994, MNRAS, 268, 173

Danziger, I. J., \& Kjaer, K. 1991, in Proc. ESO Conf., ESO/EIPC Workshop, ed. I. J. Danziger \& K. Kjaer (Garching: ESO), 37

Davenport, J., de Val-Borro, M., \& Wilkinson, T. D. 2018, PyDIS: Possibly Useful, Zenodo, doi:10.5281/zenodo.58753

Davidson, K., \& Humphreys, R. M. 2012, ASSL, 384

Davidson, K., \& Humphreys, R. M. 1997, ARA\&A, 35, 1

De, K., Tzanidakis, A., Kasliwal, M. M., Fremling, C., \& Kulkarni, S. R. 2019 ATel, 13262

de la Rosa, J., Roming, P., Pritchard, T., \& Fryer, C. 2016, ApJ, 820, 74 Fabian, A. C., \& Terlevich, R. 1996, MNRAS, 280, L5

Fassia, A., Meikle, W. P. S., Chugai, N., et al. 2001, MNRAS, 325, 907

Fassia, A., Meikle, W. P. S., Vacca, W. D., et al. 2000, MNRAS, 318, 1093

Filippenko, A. V. 1997, ARA\&A, 35, 309

Foreman-Mackey, D., Hogg, D. W., Lang, D., \& Goodman, J. 2013, PASP, 125,306

Fremling, C. 2018, TNSTR, 1463, 1

Fremling, C. 2019a, TNSTR, 414, 1

Fremling, C. 2019b, TNSTR, 586, 1

Fremling, C., Dugas, A., \& Sharma, Y. 2018a, TNSCR, 1221, 1

Fremling, C., Dugas, A., \& Sharma, Y. 2018b, TNSCR, 1870, 1

Fremling, C., Dugas, A., \& Sharma, Y. 2019a, TNSCR, 329, 1

Fremling, C., Dugas, A., \& Sharma, Y. 2019b, TNSCR, 490, 1

Fremling, C., Dugas, A., \& Sharma, Y. 2019c, TNSCR, 1

Fremling, C., \& Sharma, Y. 2018, TNSCR, 762, 1

Fremling, C., Sollerman, J., Taddia, F., et al. 2016, A\&A, 593, A68

Fremling, U. C., Miller, A. A., Sharma, Y., et al. 2019d, arXiv:1910.12973

Gall, C., Hjorth, J., Watson, D., et al. 2014, Natur, 511, 326

Gal-Yam, A. 2017, in Handbook of Supernovae, ed. A. W. Alsabti \& P. Murdin (New York: Springer), 195

Gal-Yam, A., Arcavi, I., Ofek, E. O., et al. 2014, Natur, 509, 471

Gal-Yam, A., \& Leonard, D. C. 2009, Natur, 458, 865

Gal-Yam, A., Leonard, D. C., Fox, D. B., et al. 2007, ApJ, 656, 372

Gehrels, N., Chincarini, G., Giommi, P., et al. 2004, ApJ, 611, 1005

Graham, M. J., Kulkarni, S. R., Bellm, E. C., et al. 2019, PASP, 131, 078001 
Hamuy, M., Folatelli, G., Morrell, N. I., et al. 2006, PASP, 118, 2

HEASARC 2014, HEAsoft: Unified Release of FTOOLS and XANADU, Astrophysics Source Code Library, ascl:1408.004

Hoffman, J. L., Leonard, D. C., Chornock, R., et al. 2008, ApJ, 688, 1186

Hunter, J. D. 2007, CSE, 9, 90

Kasliwal, M. M., Cannella, C., Bagdasaryan, A., et al. 2019, PASP, 131, 038003

Katz, B., Sapir, N., \& Waxman, E. 2011, arXiv:1106.1898

Khazov, D., Yaron, O., Gal-Yam, A., et al. 2016, ApJ, 818, 3

Kiewe, M., Gal-Yam, A., Arcavi, I., et al. 2012, ApJ, 744, 10

Lantz, B., Aldering, G., Antilogus, P., et al. 2004, Proc. SPIE, 5249, 146

Law, N. M., Kulkarni, S. R., Dekany, R. G., et al. 2009, PASP, 121, 1395

Leonard, D. C., Filippenko, A. V., Barth, A. J., \& Matheson, T. 2000, ApJ, 536, 239

Levesque, E. M., Stringfellow, G. S., Ginsburg, A. G., Bally, J., \& Keeney, B. A. 2014, AJ, 147, 23

Li, W.-D., Li, C., Filippenko, A. V., \& Moran, E. C. 1998, IAUC, 6829

Masci, F. J., Laher, R. R., Rusholme, B., et al. 2019, PASP, 131, 018003

Mauerhan, J., Williams, G. G., Smith, N., et al. 2014, MNRAS, 442, 1166

Murase, K., Thompson, T. A., Lacki, B. C., \& Beacom, J. F. 2011, PhRvD, 84 043003

Murase, K., Thompson, T. A., \& Ofek, E. O. 2014, MNRAS, 440, 2528

Nordin, J., Brinnel, V., Giomi, M., et al. 2019a, TNSTR, 74, 1

Nordin, J., Brinnel, V., Giomi, M., et al. 2019b, TNSTR, 464, 1

Nordin, J., Brinnel, V., Giomi, M., et al. 2019c, TNSTR, 502, 1

Nyholm, A., Sollerman, J., Tartaglia, L., et al. 2020, A\&A, 637, 73

Ofek, E. O. 2014, MATLAB package for astronomy and astrophysics, Astrophysics Source Code Library, ascl:1407.005

Ofek, E. O., Fox, D., Cenko, S. B., et al. 2013a, ApJ, 763, 42

Ofek, E. O., Rabinak, I., Neill, J. D., et al. 2010, ApJ, 724, 1396

Ofek, E. O., Sullivan, M., Cenko, S. B., et al. 2013b, Natur, 494, 65

Ofek, E. O., Zoglauer, A., Boggs, S. E., et al. 2014, ApJ, 781, 42

Oke, J. B., Cohen, J. G., Carr, M., et al. 1995, PASP, 107, 375

Patat, F., Taubenberger, S., Benetti, S., Pastorello, A., \& Harutyunyan, A. 2011, A\&A, 527, L6

Patterson, M. T., Bellm, E. C., Rusholme, B., et al. 2019, PASP, 131, 018001

Payne, A. V., Tucker, M. A., Do, A., Shappee, B. J., \& Huber, M. E. 2019, TNSCR, 97, 1

Perley, D. A. 2019, PASP, 131, 084503

Poole, T. S., Breeveld, A. A., Page, M. J., et al. 2008, MNRAS, 383, 627

Prentice, S. J., Maguire, K., Magee, M. R., Clark, P., \& Skillen, K. 2018, TNSCR, 1952, 1
Prentice, S. J., Maguire, K., Skillen, K., Magee, M. R., \& Clark, P. 2019, TNSCR, 602, 1

Rahmer, G., Smith, R., Velur, V., et al. 2008, Proc. SPIE, 7014, 70144Y

Rau, A., Kulkarni, S. R., Law, N. M., et al. 2009, PASP, 121, 1334

Reilly, E., Maund, J. R., Baade, D., et al. 2017, MNRAS, 470, 1491

Richardson, D., Jenkins, R. L., Wright, I., Jr., \& Maddox, L. 2014, AJ, 147,118

Rigault, M., Neill, J. D., Blagorodnova, N., et al. 2019, A\&A, 627, A115

Roming, P. W. A., Kennedy, T. E., Mason, K. O., et al. 2005, SSRv, 120, 95

Sagiv, I., Gal-Yam, A., Ofek, E. O., et al. 2014, AJ, 147, 79

Schlafly, E. F., \& Finkbeiner, D. P. 2011, ApJ, 737, 103

Schlegel, E. M. 1990, MNRAS, 244, 269

Schlegel, E. M., \& Petre, R. 2006, ApJ, 646, 378

Smith, N. 2014, ARA\&A, 52, 487

Smith, N., Chornock, R., Li, W., et al. 2008, ApJ, 686, 467

Smith, N., Kilpatrick, C. D., Mauerhan, J. C., et al. 2017, MNRAS, 466, 3021

Smith, N., Mauerhan, J. C., \& Prieto, J. L. 2014, MNRAS, 438, 1191

Soumagnac, M. T., Ganot, N., Gal-yam, A., et al. 2019a, arXiv:1907. 11252

Soumagnac, M. T., Ofek, E. O., Gal-yam, A., et al. 2019b, ApJ, 872, 141

Stathakis, R. A., \& Sadler, E. M. 1991, MNRAS, 250, 786

Steele, I. A., Smith, R. J., Rees, P. C., et al. 2004, Proc. SPIE, 5489, 679

Stoll, R., Prieto, J. L., Stanek, K. Z., et al. 2011, ApJ, 730, 34

Taddia, F., Stritzinger, M. D., Sollerman, J., et al. 2013, A\&A, 555, A10

Tody, D. 1986, Proc. SPIE, 627, 733

Tody, D. 1993, in ASP Conf. Ser. 52, IRAF in the Nineties, ed. R. J. Hanisch, R. J. V. Brissenden, \& J. Barnes (San Francisco, CA: ASP), 173

Tonry, J., Stalder, B., Denneau, L., et al. 2018a, TNSTR, 695, 1

Tonry, J., Stalder, B., Denneau, L., et al. 2018b, TRNSTR, 1186, 1

Tucker, M. A., Payne, A. V., Do, A., Huber, M. E., \& Shappee, B. J. 2019, TNSCR, 514, 1

Turatto, M., Cappellaro, E., Danziger, I. J., et al. 1993, MNRAS, 262, 128

Valenti, S., Sand, D., Pastorello, A., et al. 2014, MNRAS, 438, L101

van Dyk, S. D., Weiler, K. W., Sramek, R. A., \& Panagia, N. 1993, ApJL, 419, L69

Virtanen, P., Gommers, R., Oliphant, T. E., et al. 2020, Nature Methods, 17,261

Wang, L., \& Wheeler, J. C. 2008, ARA\&A, 46, 433

Williams, C. L., Panagia, N., Van Dyk, S. D., et al. 2002, ApJ, 581, 396

Yaron, O., Perley, D. A., Gal-Yam, A., et al. 2017, NatPh, 13, 510

Zackay, B., Ofek, E. O., \& Gal-Yam, A. 2016, ApJ, 830, 27 